\title{
A unique demographic history exists for the $M A O-A$ gene in Polynesians
}

\author{
David A Eccles ${ }^{1,2}$, Donia Macartney-Coxson ${ }^{2}$, Geoffrey K Chambers ${ }^{1}$ and Rodney A Lea ${ }^{2,3}$
}

Variation in the human monoamine oxidase $A(M A O-A)$ gene can influence neurotransmittor levels and is thought to have a role in many behavioral traits. The genetic architecture of $M A O-A$ is known to vary across different geographic subgroups. Previous studies have reported evidence for positive selection within the MAO-A gene region in seven ethnic groups: Pygmy, Aboriginal Taiwanese, Chinese, Japanese, Mexican and Russian. Polynesian populations have not been tested and repeated founder effects due to the island-hopping voyages of Polynesians across the South Pacific suggest a unique demographic history exists at the MAO-A gene, perhaps including selective effects. To explore this, we genotyped 13 key single-nucleotide polymorphisms (SNPs) spanning MAO-A gene as well as the functional polymorphism (MAO-A-uVNTR) in 47 unrelated Maori individuals. A comparison of genetic variation between Maori and non-Maori groups found a substantial reduction in genetic diversity at the $M A O-A$ gene locus and an increase in the frequency of the most common MAO-A gene variant in the Maori group. Results of this study support previous findings and also point toward a 5-SNP haplotype that may have been influenced by selective effects in the Maori population. Full-sequence data for $M A O-A$ in a large cohort are now required to conclusively determine whether $M A O-A$ has undergone positive selection in Polynesians. Overall, these new data describe a unique demographic history for the $M A O-A$ gene in the Maori population and will be helpful for studies wishing to investigate $M A O-A$ as a candidate gene for influencing behavioral traits in the Polynesians.

Journal of Human Genetics (2012) 57, 294-300; doi:10.1038/jhg.2012.19; published online 1 March 2012

Keywords: genotypes; haplotypes; MAO-A; Maori; mutation; VNTR

\section{INTRODUCTION}

Monoamine oxidases (MAO, EC 1. 4.3.4) are flavoenzymes that are bound to the outer mitochondrial membrane. The two known MAO proteins are encoded by two separate (but closely linked) genes, monoamine oxidase $A(M A O-A)$ and monoamine oxidase $B(M A O B)$. They are found almost tail-to-tail (20-kb separation) on the $\mathrm{X}$ chromosome (region $\mathrm{Xp} 11.3$ ), and are $70 \%$ identical at the aminoacid levels. ${ }^{1}$ In the promoter sequence $1.2-\mathrm{kb}$ upstream of the MAO-A gene, there is a variable-number tandem repeat polymorphism (VNTR) of a 30-base pair repeat sequence element (MAO-AuVNTR) that is known to influence gene expression levels. ${ }^{2}$ The 3- and 5-repeat variants result in low expression, whereas the 3.5- and 4-repeat variants result in high expression. High and low expression variants occur at different frequencies in different ethnic groups (Supplementary Figure 1). This promoter polymorphism has been found to be in linkage disequilibrium (LD) with genetic markers within the MAO-A gene. ${ }^{3}$

Gilad et al. ${ }^{4}$ carried out a study of nucleotide diversity at the $M A O-A$ gene region in humans and discovered extensive variation across seven different ethnic groups: Ashkenazi, Pygmy, Aboriginal
Taiwanese, Chinese, Japanese, Mexican and Russian. A direct sequencing approach was used in order to ensure a high-resolution analysis of total nucleotide variation across the region. A total of five segments of the gene were selected with a total combined length of $18.8 \mathrm{~kb}$, comprising about $20 \%$ of the entire $90-\mathrm{kb}$ gene region. A total of 41 polymorphic sites were observed: 33 single-nucleotide polymorphisms (SNPs), 7 deletions and the MAO-A-uVNTR. The polymorphic status of mutations was not consistent across all groups. Only 12 of 41 sites were polymorphic in all groups genotyped. ${ }^{4}$

Based on their observations of LD patterns across the MAO-A gene region, and reduced diversity within groups, Gilad et al. ${ }^{4}$ suggested that there may have been positive selection in this region acting on $M A O-A$-related phenotypes. The most obvious evidence for selection was consistently higher LD than that expected under a neutral recombination model. Supporting evidence for positive selection was provided by the observation of low within-group diversity combined with high between-group diversity. ${ }^{4}$

The study carried out by Gilad et al. ${ }^{4}$ did not include Polynesian individuals, but Polynesian populations have a close genetic similarity to tribes from Island South-East Asia, which further corroborates

${ }^{1}$ School of Biological Sciences, Victoria University of Wellington, Wellington, New Zealand; ${ }^{2}$ Department of Population and Environmental Health, Institute of Environmental Science and Research Limited, Porirua, New Zealand and ${ }^{3}$ Department of Computational Genomics and Medicine, Genomics Research Centre, School of Medical Sciences, Griffith Health Institute, Griffith University, Queensland, Australia

Correspondence: Dr R Lea, Department of Computational Genomics and Medicine, Genomics Research Centre, School of Medical Sciences, Griffith Health Institute, Griffith University, Gold Coast Campus, Queensland 4222, Australia.

E-mail: r.lea@griffith.edu.au

Received 8 August 2011; revised 9 January 2012; accepted 10 January 2012; published online 1 March 2012 
evidence from linguistic and cultural research that suggests a Taiwanese origin. ${ }^{5}$ Therefore, an obvious extension for research on positive selection at the $M A O-A$ gene is to investigate selection at this genomic region in a Polynesian population. The native Maori population of New Zealand is believed to descend from island-hopping adventurers from Eastern Polynesia, who traveled to New Zealand around $600-800$ years ago. 6,7

The current study is a natural extension of the work of Gilad et al. ${ }^{4}$ with the goal of investigating the demographic history of the MAO-A gene in the Maori population for the purpose of testing for positive selection and designing better-informed candidate gene association studies of behavioral traits.

\section{MATERIALS AND METHODS}

This study has an intra-population and inter-population design, looking at haplotype block patterns in the neighborhood of the MAO-A gene region. Haplotypes were scored in males and females using banked DNA samples selected from the New Zealand Maori population described in Hall et al. ${ }^{8}$

\section{Variants typed}

All references to locations of genes and mutations are based on the NCBI reference assembly for Homo sapiens, build 36, 21 March 2006. Gene names are the same as those in the NCBI database as of May 2007.

The genomic sequence for the $M A O-A$ gene, including $10 \mathrm{~kb}$ of flanking sequence (both upstream and downstream), was also retrieved from the NCBI database (NC_000023:433390353-43501012_rev). Flanking sequences for MAO-A-uVNTR and 13 of the SNPs typed by Gilad et al. ${ }^{4}$ were requested from the authors (Figure 1), and located within the retrieved MAO-A genomic sequence (see Table 1 for mutations and flanking sequences).

All 13 SNP positions were confirmed as present within the MAO-A gene using the ssearch program, http://fasta.bioch.virginia.edu/fasta_www2/fasta list2.shtml. ${ }^{9}$ Hits within chromosome $\mathrm{X}$ were found for all SNPs except 2.3(476). After carrying out this process, it was noticed that one SNP, 1.1(635), resides within the 30 -base pair repeat sequence.

\section{Study groups}

Genotyping was done via a service contract with the Australian Genome Research Facility (AGRF, http://www.agrf.org.au), using the Sequenom MassArray Genotyping system. ${ }^{10}$ The 13 SNPs, plus the 30-bp-repeat VNTR, were typed in 47 unrelated Maori individuals with a self-reported ancestry of four Maori grandparents (the same 47 individuals typed in Hall et al. ${ }^{8}$ ).
Genotype data were analyzed using Haploview ${ }^{11}$ to establish whether particular combinations of SNPs could be treated as a single haplotype block. ${ }^{11}$ Female haplotype counts were determined from unphased data using the method of Clark, ${ }^{12}$ assuming that a haplotype was present in an individual if a previously observed haplotype could generate the observed unphased genotype data. ${ }^{12}$ The significance of haplotype and VNTR frequency differences between Maori and other groups was determined using the $\chi^{2}$ test function of $\mathrm{R}$ (R Development Core Team, Vienna, Australia, 2008), treating the total Gilad et al. ${ }^{4}$ data as an expected probability, and simulating the $\chi^{2}$ distribution when genotype counts for either Maori or all Gilad et al. ${ }^{4}$ groups were below 5 .

To give an accurate comparison of results, neutrality tests carried out by Gilad et al. ${ }^{4}$ were repeated, restricting analysis to the subset of 11 successfully genotyped SNPs, and including genotype data from Maori males. The statistical tests of neutrality were carried out using the program VariScan version 2.0.2, http://www.ub.es/softevol/variscan.

The neutrality tests were restricted to those that did not require fullsequence data in order to provide an accurate result. The sensitivity of neutrality tests to sequence changes was determined by embedding short

Table 1 Mutations typed in the MAO-A gene region

\begin{tabular}{ll}
\hline Mutation & \multicolumn{1}{c}{ Sequence $5^{\prime}-3^{\prime}$} \\
\hline $1.1(263)$ & AGAGCCCACGCGGCTACACCCACRTCTACTCCCCCACTCTC \\
$1.1(635)$ & ACCAGTACCGGCACCRGCACCAGTACCCGCACCAGTACCGG \\
VNTR & ACAGCCTGACCGTGGAGAAG... TCCGAATGGAGCGTCCGTTC \\
-(repeat) & ((ACCGGC)(ACCGGC)(ACCAGT)(ACCCGC)(ACCAGT)) \\
$1.2(769)$ & CAAAAGGGTTCGCCCCGCSCACAGTGCCCGGCTCCCCCCGG \\
$1.5(617)$ & AGTGATCTACAACCATAMTGCTTTTAGGAGGCTTGCCTAGT \\
$2.2(163)$ & GAGTTGCTGAGAAGCAGGTTTTTYAGCATGGAGATAAAGAA \\
$2.3(476)$ & AGAATTGCTTGAACCCRGGAGGCGGAGCTTGCAGTGAGCCG \\
$2.4(427)$ & GTGTAGGCTATGCATAGYCTTTTACAGTATGTTAAGATGGG \\
$3.1(224)$ & TTACATGGATCATTYAACAAAATAATATATAGCCAGCAAT \\
$3.4(166)$ & TATCACAGTGTCTGGGRGGTGTGGCCCTGCCCCCTACTAC \\
$5.1(183)$ & GACAACTATTTCTAGAATTTCAYTGAACTCTGCTTTCCT \\
$5.1(555)$ & GTATACTTTGCTCTTMCCATTTTCTTGATTAGGGAAGACAT \\
$5.4(790)$ & TCAGGTTCTTGTACCCAGATRTCTTTCTCGGTCACCTTCCC \\
$5.6(776)$ & ACACCAGGGTCCAGCAMCTTAGGTTTGAATTTATGATAAGG
\end{tabular}

Abbreviations: $M A O-A$, monoamine oxidase $A$; VNTR, variable-number tandem repeat polymorphism.

Nomenclature indicates segment name and sequence position () within each region for the mutation as per Gilad et al ${ }^{4} \mathrm{M}, \mathrm{R}, \mathrm{S}$ and $\mathrm{Y}$ (given in bold) indicate nucleotide polymorphisms $A / C, A / G, C / G$ and $C / T$, respectively.

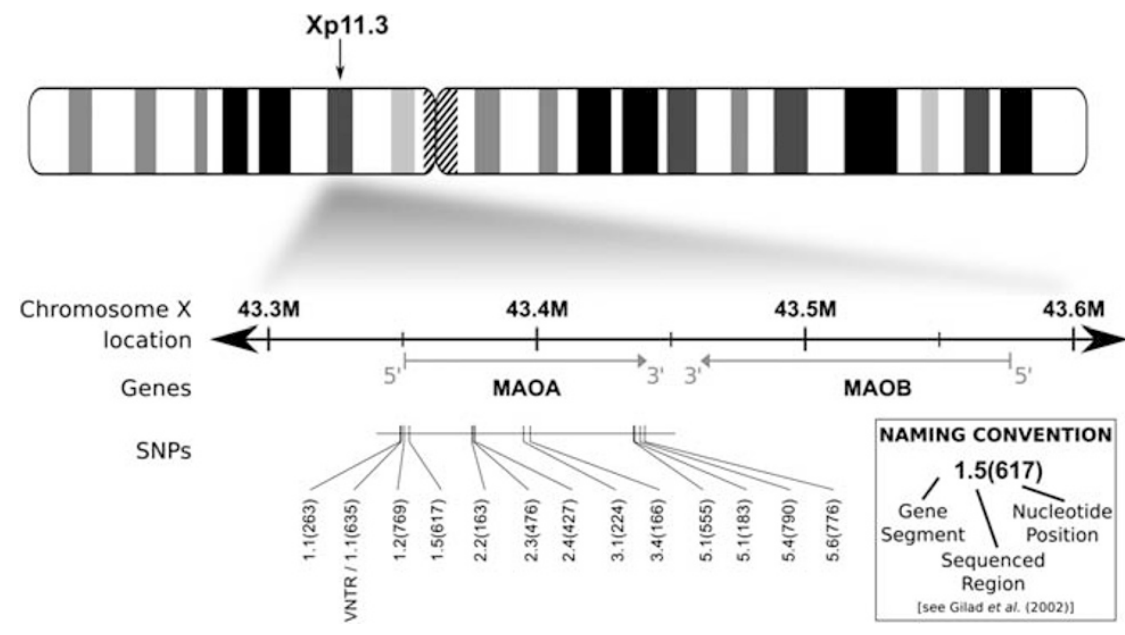

Figure 1 Section of chromosome $\mathrm{X}$, showing the relative positions of the MAO-A and MAOB genes, and mutations typed in this study. Labels for each mutation refer to the segment, region and nucleotide position as per Gilad et al. ${ }^{4}$ 
stretches of non-polymorphic pseudo-sequence between polymorphic sites. Statistics were excluded when the calculated statistic with added pseudosequence differed from that when pseudo-sequence was absent. Fu and Li's $D$ statistic ${ }^{13}$ was also excluded because although it was present in their population variability parameter table, the statistic was not discussed by Gilad et al. ${ }^{4}$ The $r^{2}$ statistic was included to supplement the other recombination statistic, $D^{\prime}$.

\section{$\mathrm{X}$-chromosome inactivation}

The $M A O-A$ gene resides on the $\mathrm{X}$ chromosome, so care must be taken in the interpretation of association study results in females. Inactivation of one of the two X chromosomes during early development results in a mosaic of phenotypes across all the cells of the body. Although some X-chromosome genes can escape inactivation, MAO-A has been found to be monoallelic in skin fibroblasts, ${ }^{14}$ so one copy is likely to be inactivated. ${ }^{14}$

Females who are homozygous for a particular genetic variant will have that same variant in all cells, regardless of which chromosome is inactivated, so can usually be considered to have a similar genetic profile to hemizygous males (although statistical tests should be carried out first in order to confirm that male and female data can be combined). However, the nature of the $\mathrm{X}$-chromosome inactivation process can mean that it is difficult to know which variant may be active in the particular cells of interest for a heterozygous female. Owing to these mosaic effects, it is probably best to remove $\mathrm{X}$-chromosome data for heterozygotes from association studies, because inclusion of such data could generate false results.

However, a removal of heterozygous data is not necessary for investigations of genetic patterns and chromosomal recombination, as in the current study. These types of analyses aim to determine the history of a variant, rather than its current effect. Mosaic effects from X-chromosome inactivation in females will not be expected to affect the outcome of the study, because this study just counts alleles.

\section{RESULTS}

Polymorphism in the Maori group

Of the 13 SNPs analyzed (all polymorphic in at least 1/7 groups genotyped by Gilad et al. ${ }^{4}$ ), 2 were unable to be genotyped (1.1(635) and 2.3(476)) and 5/11 were found to be polymorphic in the Maori group (1.1(263), 1.5(617), 3.1(224), 5.1(183) and 5.4(790): Table 2, Supplementary Figure 2). The MAO-A-uVNTR was also polymorphic in the Maori group. For the remaining six SNPs, all individuals had the most common variant (with respect to other groups) at each site.

\section{Full LD among all SNPs}

Genotype data from Maori females were analyzed using Haploview (Figure 2), revealing complete LD between all SNPs $\left(D^{\prime}=1\right)$, but incomplete LD between all SNPs and the VNTR $\left(D^{\prime}=0.66\right)$. Only three SNP haplotypes were observed within the $M A O-A$ region in Maori females (AGCCG, GATTA and AGTTA), out of a possible 32 that would be expected for five unlinked dimorphic SNPs.

\section{Haplotype counts for the $M A O-A$ gene}

Given that LD was found to be complete for all five polymorphic SNPs across the entire $M A O-A$ gene region, these SNPs were combined into a single haplotype. Table 3 summarizes the count data recorded for these polymorphisms in all genotyped individuals, including data from Gilad et al. ${ }^{4}$ Counts for heterozygous 3/4 females appear separately in the table ( 7 females had a heterozygous VNTR genotype that could not be assigned with certainty to a particular chromosome).

Only 2 MAO-A 5-SNP haplotypes were observed in the 18 Maori males that were genotyped: 14 AGCCG haplotypes and 4 GATTA haplotypes. These two haplotypes are the most common globally, and differ at all sites. As males have only one $\mathrm{X}$ chromosome, these haplotypes can be inferred directly from the genotypes. Among the
Table 2 Genotyping overview indicating the polymorphic status of mutations typed in the Maori group

\begin{tabular}{|c|c|c|c|c|c|}
\hline \multirow[b]{2}{*}{ Locus } & \multirow[b]{2}{*}{ Typed } & \multicolumn{2}{|c|}{ Variants } & \multicolumn{2}{|c|}{ Polymorphic a } \\
\hline & & Common & Rare & All & Maori \\
\hline $1.1(263)$ & $Y$ & A & G & $Y$ & $Y$ \\
\hline $1.1(635)$ & $\mathrm{N}$ & $\mathrm{G}$ & A & $\mathrm{N}$ & - \\
\hline uVNTR & $Y$ & $3^{b}$ & 4 & Y & $Y$ \\
\hline $1.2(769)$ & $Y$ & C & $\mathrm{G}$ & $\mathrm{N}$ & $\mathrm{N}$ \\
\hline $1.5(617)$ & Y & C & $\mathrm{T}$ & Y & Y \\
\hline $2.2(163)$ & $Y$ & $\mathrm{~T}$ & C & $\mathrm{N}$ & $\mathrm{N}$ \\
\hline $2.3(476)$ & $\mathrm{N}$ & A & G & Y & - \\
\hline $2.4(427)$ & Y & C & $\mathrm{T}$ & $\mathrm{N}$ & $\mathrm{N}$ \\
\hline $3.1(224)$ & Y & C & $\mathrm{T}$ & Y & Y \\
\hline $3.4(166)$ & $Y$ & A & G & $\mathrm{N}$ & $\mathrm{N}$ \\
\hline $5.1(183)$ & $Y$ & C & $\mathrm{T}$ & Y & Y \\
\hline $5.1(555)$ & $Y$ & C & A & $\mathrm{N}$ & $\mathrm{N}$ \\
\hline $5.4(790)$ & $Y$ & $\mathrm{G}$ & $A$ & Y & $Y$ \\
\hline $5.6(776)$ & $Y$ & A & C & $\mathrm{N}$ & $\mathrm{N}$ \\
\hline
\end{tabular}

aIndicates whether a mutation was polymorphic in all groups typed by Gilad et al., 'All', and whether it was polymorphic in the Maori group, 'Maori'.

bThe common variant globally for MAO-A-UVNTR is a sequence repeated four times, whereas the common variant in Maori is a sequence repeated three times (globally rare). For all other mutations, the common variant in Maori was consistent with the common variant in other groups.

Two mutations, 1.1(635) and 2.3(476), were unable to be genotyped.
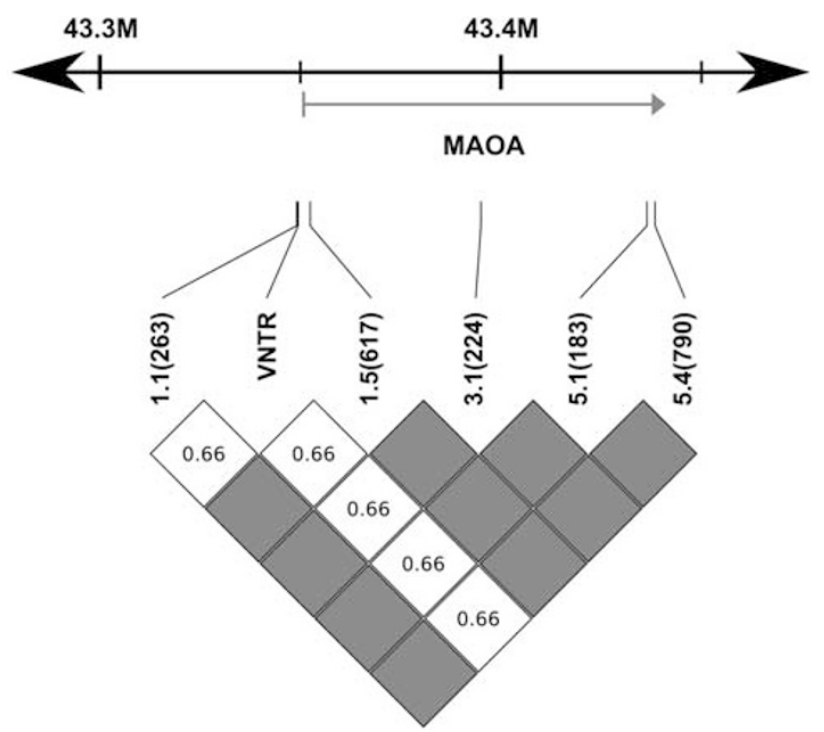

Figure 2 Haploview diagram showing full LD in the Maori group across the MAO-A gene. Grey diamonds indicate full LD between the two SNPs. Numbers inside squares represent $D^{\prime}, D^{\prime}$ is 1 where not specified. The VNTR was treated as a dimorphism and included in this Haploview analysis.

29 female haplotypes that were genotyped across the MAO-A region, three different haplotypes were observed: 45 AGCCG, 12 GATTA and 1 AGTTA haplotype. These haplotype counts do not differ significantly from those for Maori males $\left(\chi^{2}=0.33, P>0.8\right)$. There were 10 heterozygous females across this gene region for both the AGCCG and GATTA haplotypes; another female was found to be heterozygous with an AGTTA and AGCCG combined haplotype.

The 3-repeat VNTR variant was observed in 10 Maori males and in 9 Maori females (only 2 females were homozygous for this variant, the 
Table 3 Proportions of VNTR variants for SNP haplotypes observed in the MAO-A gene region for 18 Maori males, 56 non-Maori males (from Gilad et al. ${ }^{4}$ ) and 29 Maori females

$\begin{array}{lccccc}\text { Maori male VNTR proportions } & & & & \\ \text { 5-SNP haplotype } & 3 & 4 & \text { NG } & n & \text { pHT } \\ \text { AGCCG } & 0.50 & 0.50 & 2 & 14 & 0.78 \\ \text { GATTA } & 1.00 & 0.00 & 0 & 4 & 0.22 \\ \text { Total } & 0.62(10) & 0.38(6) & 2 & 18 & 1.00\end{array}$

\begin{tabular}{|c|c|c|c|c|c|c|}
\hline 5-SNP haplotype & 3 & & 4 & $N G$ & $n$ & $\mathrm{pHT}$ \\
\hline AGCCG & 0.17 & & 0.83 & 0 & 23 & 0.41 \\
\hline GATTA & 1.00 & & 0.00 & 0 & 10 & 0.18 \\
\hline GGCCG & 0.00 & & 1.00 & 0 & 7 & 0.12 \\
\hline GACTA & 0.67 & & 0.33 & 0 & 3 & 0.05 \\
\hline GGCTA & 1.00 & & 0.00 & 0 & 2 & 0.04 \\
\hline AGCTG & 0.50 & & 0.50 & 0 & 2 & 0.04 \\
\hline AACCG & 0.00 & & 1.00 & 0 & 2 & 0.04 \\
\hline AGCTA & 0.00 & & 1.00 & 0 & 1 & 0.02 \\
\hline GACCG & 1.00 & & 0.00 & 0 & 1 & 0.02 \\
\hline GACCA & 1.00 & & 0.00 & 0 & 1 & 0.02 \\
\hline AACTA & 1.00 & & 0.00 & 0 & 1 & 0.02 \\
\hline AGTCG & 1.00 & & 0.00 & 0 & 1 & 0.02 \\
\hline GGTTA & 1.00 & & 0.00 & 0 & 1 & 0.02 \\
\hline GATTG & 1.00 & & 0.00 & 0 & 1 & 0.02 \\
\hline Total & $0.45(25)$ & & $0.55(31)$ & 0 & 56 & 1.00 \\
\hline \multicolumn{7}{|c|}{ Maori female VNTR proportions } \\
\hline 5-SNP haplotype & 3 & $3 / 4$ & 4 & $N G$ & $\mathrm{n}$ & $\mathrm{pHT}$ \\
\hline AGCCG & 0.14 & 0.00 & 0.86 & 3 & 17 & 0.59 \\
\hline AGCCG/GATTA & 0.00 & 1.00 & 0.00 & 3 & 10 & 0.34 \\
\hline AGCCG/AGTTA & - & - & - & 1 & 1 & 0.03 \\
\hline GATTA & 0.00 & 0.00 & 1.00 & 0 & 1 & 0.03 \\
\hline Total & $0.09(2)$ & $0.32(7)$ & $0.59(13)$ & 7 & 29 & 1.00 \\
\hline
\end{tabular}

Abbrevaitions: MAO-A, monoamine oxidase A; SNP, single-nucleotide polymorphism; VNTR, variable-number tandem repeat polymorphism.

$n$, total number of individuals with this SNP haplotype; NG, number of individuals who could not be genotyped for any VNTR variant; $\mathrm{pHT}$, proportion of this SNP haplotype in the group.

remaining 7 were heterozygous, Table 3 ). The 4-repeat VNTR variant was observed in 6 males and 20 females (33 4-repeat VNTR variants were observed in females). The proportion of haplotypes with the 3 -repeat VNTR was significantly higher in males (0.62) than in females $\left(0.09, \chi^{2}=12, P<0.003\right)$.

In all cases where a $3 / 4$ heterozygote VNTR genotype was observed, both the rare and common SNP haplotypes were also observed (AGCCG and GATTA, respectively). No male was observed with the GATTA haplotype combined with the 4-repeat VNTR, but one female was observed with two copies of the GATTA haplotype and was also homozygous for the 4-repeat VNTR.

\section{$M A O-A$ region haplotype frequency comparisons between Maori and non-Maori groups}

Haplotypes were initially dichotomized into 'common' (AGCCG) and 'not common' (any other haplotype) in an attempt to better compare Maori with other ethnic groups (Supplementary Figure 3). The proportion of the most common SNP haplotype (AGCCG) is greater in Maori males (0.78) than in non-Maori males $\left(0.41, \chi^{2}=10.02\right.$, $P=0.0015)$. Of those individuals who have the higher frequency AGCCG haplotype, a smaller proportion of Maori (0.50) have the 4-repeat VNTR allele than non-Maori group $\left(0.83, \chi^{2}=10.36\right.$, $P<0.008)$.

Figure 3 shows a mutation network diagram for the 5-SNP MAO-A haplotypes that were found. The figure can be broken into two parts comprising mutations likely to be related to each of the two most common haplotypes, AGCCG and GATTA, separated by dotted lines. While GGCCG was the third most common haplotype observed by Gilad et al., ${ }^{4}$ only two haplotypes were found that differed from it at a single locus, AGCCG (the most common haplotype) and GACCG. The common 4-repeat VNTR polymorphism was not found at all in conjunction with the lower-frequency GATTA SNP haplotype. Only one GATTA-related haplotype, GACTA, was found in conjunction with the common 4-repeat VNTR allele. However, the 3-repeat allele was found in conjunction with three AGCCG-related haplotypes (AGCCG, AGTCG and AGCTG).

The frequency difference for the common haplotype between Maori and non-Maori groups is similar when combining counts for singlemutation variants of the most common haplotype (GGCCG, AACCG, AGTCG and AGCTG). When including these variants, the proportion of non-Maori groups with common variants is $0.625\left(\chi^{2}=1.7926\right.$, $P=0.18)$. The novel AGTTA haplotype found in a single female chromosome (Table 3) was not present in any of the haplotypes reported by Gilad et al. ${ }^{4}$

\section{Reanalysis of neutrality tests}

For direct comparison with Gilad et al., ${ }^{4}$ neutrality tests were repeated in the Maori male subgroup, restricting analysis to the 11 SNPs genotyped in Maori (Table 4). Statistics that required full-sequence data were excluded because they could not be calculated for this group.

Most statistics calculated for Maori males lay within the ranges observed for the male groups genotyped by Gilad et al. ${ }^{4}$ However, there were two clear outliers: the number of distinct haplotypes $(\mathrm{K})$ and $r^{2}$. Despite having the largest group sample size, the Maori sample had the lowest number of observed haplotypes indicating a substantial reduction in genetic diversity across the $M A O-A$ gene. Importantly, the $r^{2}$ statistic, an index of $\mathrm{LD}$, was highest in the Maori group reflecting the extended haplotypic regions in this population. The Tajima's $D$ statistic for Maori is also quite high relative to others, which is indicative of non-neutrality perhaps due to selection.

\section{DISCUSSION}

This study has identified three distinct haplotypes in Maori (18 males and 29 females) across a 90-kb region encompassing the MAO-A gene. Haplotypes for males were determined directly from genotype data for the $\mathrm{X}$ chromosome; the low number of haplotypes observed in all Maori allowed haplotypes to also be determined for females.

Of the statistical tests that were compared between groups typed by Gilad et al. ${ }^{4}$ and the Maori male subgroup, two are clear outliers in Maori, as indicated by a high $r^{2}$ and low $\mathrm{K}$ (Table 4). The values of both these statistics indicate a substantially reduced variation in MAO$A$ gene diversity in the Maori population. Other statistics lie within the range of what has been observed in non-Maori groups.

Only 2 out of 32 possible SNP haplotypes were identified in Maori males across the entire 90-kb region, whereas 14 different haplotypes were observed in non-Maori males (Table 3). While the two MAO-A haplotypes found in Maori are also the most common haplotypes found in non-Maori groups, they differ in overall proportion between the two groups. One possible recombinant haplotype was observed (AGTTA, Table 3). While sequence coverage was greater in the Gilad et $a{ }^{4}{ }^{4}$ study, the Maori data suggest that three assays should be a 

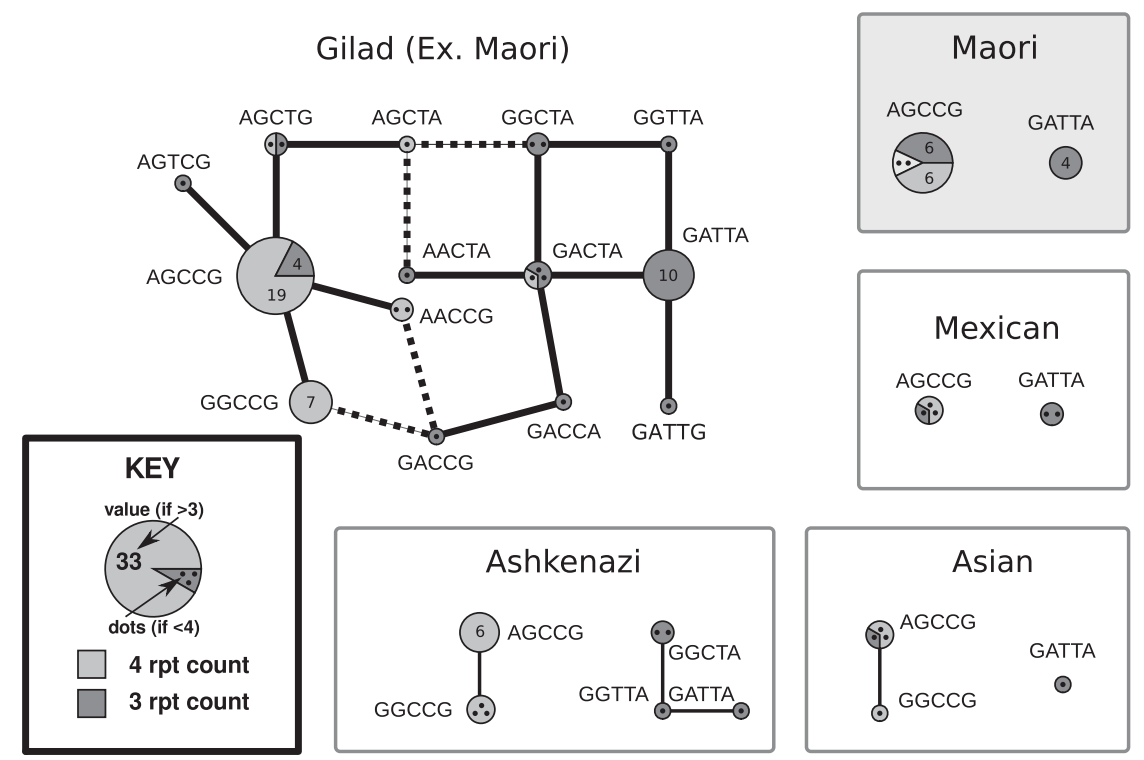

Figure 3 The haplotype mutation network for the MAO-A gene region showing observed 5-SNP MAO-A haplotypes in male individuals for all Gilad et al. ${ }^{4}$ groups combined, as well as Maori, Mexican, Japanese/Chinese and Ashkenazi groups. The SNP haplotypes are indicated by labels, and VNTR variants are indicated by colored segments; the common 4-repeat VNTR variant is light grey, the rare 3-repeat variant is dark grey and not-genotyped is white. Area of the circles is proportional to the number of haplotypes found (also indicated by numbers, or by dots for fewer than four samples). Lines indicate all single mutations that would change from one observed haplotype to another. Dotted lines require both a SNP and a VNTR mutation to be consistent with observed haplotypes.

Table 4 Neutrality tests of the MAO-A gene region in the Gilad et al. ${ }^{4}$ and Maori groups for 11 SNPs genotyped in Maori

\begin{tabular}{lrrrrrr}
\hline Group & $\mathrm{N}$ & $\mathrm{S}$ & $\mathrm{K}$ & $\mathrm{D}^{\prime}$ & $\mathrm{r}^{2}$ & Tajima's D \\
\hline Ashkenazi & 13 & 8 & 7 & 1 & 0.187 & -0.329 \\
Bedouin & 10 & 6 & 6 & 0.958 & 0.262 & 0.544 \\
Pygmy & 7 & 9 & 6 & 0.893 & 0.224 & 0.444 \\
Taiwan & 9 & 5 & 7 & 0.805 & 0.315 & 1.520 \\
Japanese/Chinese & 5 & 6 & 4 & 1 & 0.528 & -0.668 \\
Mexican & 5 & 8 & 4 & 0.851 & 0.488 & 1.028 \\
Russian & 7 & 8 & 6 & 0.854 & 0.255 & 0.722 \\
Total (Gilad et al. ${ }^{4}$ ) & 56 & 11 & 23 & 0.762 & 0.105 & 0.630 \\
Maori & 18 & 5 & 2 & 1 & 1 & 0.820 \\
\hline
\end{tabular}

Abbrevaitions: MAO-A, monoamine oxidase A; SNP, single-nucleotide polymorphism. $N$ number of individuals genotyped. $S$, number of polymorphic sites; and $K$, number of , hserved distinct haplotypes. The $D^{\prime}$ and $r^{2}$ statistics are mean linkage values across the entir gene region. Statistics used by Gilad et al. ${ }^{4}$ and sensitive to sequence data $(\theta, \pi$ and $H)$ have been excluded due to the absence of relevant sequence data for our samples.

suitable proxy for sequencing the entire genome in Maori: two SNPs together with the VNTR polymorphism. The number of genotyped Maori individuals was chosen to be comparable in size to that in the study carried out by Gilad et al., ${ }^{4}$ and, as an individual group, exceeds the number of individuals typed for all other populations at this region (Table 4). Given the observation of substantial variation in other groups, variation beyond the two core haplotypes should have been seen in Maori if it existed in substantial proportions of the population. However, this observation should be validated in a larger cohort of Maori.

\section{Large haplotype block size in Maori}

The Haploview analysis indicated that all SNPs within the 90-kb $M A O-A$ gene region were tightly linked in Maori (Figure 2). This complete linkage is a feature of the Maori group that was not observed in any of the groups analyzed by Gilad et al. ${ }^{4}$ (Table 4 ). The most similar group (with respect to linkage across the entire region) was a combined Chinese and Japanese group $\left(D^{\prime}=1, r^{2}=0.528, n=5\right)$. The Maori group described here was only genotyped across a small section of the MAO-A gene region; in a future study, it would be helpful to analyze genotype data across a larger region to confirm that $\mathrm{LD}$ extends over a greater region than the MAO-A gene itself.

Gabriel et al. ${ }^{15}$ carried out a study that identified haplotype blocks in 51 autosomal regions covering $13 \mathrm{Mb}$ of sequence dispersed throughout the human genome, with an average size of $250 \mathrm{~kb}$ per region. Their study identified blocks in African-American genomes of up to $94 \mathrm{~kb}$ in length (mean length $9 \mathrm{~kb}$ ) and up to $173 \mathrm{~kb}$ in length (mean length $18 \mathrm{~kb}$ ) in European and Japanese/Chinese genomes. Considering the SNPs within the MAO-A region, the block length of $90 \mathrm{~kb}$ identified here (Figure 2) lies within these ranges. Recombination in the $\mathrm{X}$ chromosome occurs less frequently than in the autosomes, so a 90-kb haplotype block may not necessarily represent a 'large' unit on this chromosome. ${ }^{15}$ Although Gilad et al. ${ }^{4}$ mentioned that the neighborhood of the $M A O-A$ region experiences high recombination (up to $4: 58 \mathrm{cM} \mathrm{Mb}^{-1}$ ), they observed no decay of $\mathrm{LD}$ across the $90-\mathrm{kb}$ region identified here. ${ }^{4}$ Our results are similar to Gilad et al. ${ }^{4}$ with respect to LD decay (or lack of it), but differ in that a greater proportion (that is, 100\%) of the SNPs show complete LD in the Maori group.

Recombination across the MAO-A gene is likely to be longer than $90 \mathrm{~kb}$ and further genotyping of the region surrounding the MAO-A gene is required to establish the full extent of this block. In fact, an unpublished study has found that this block spans almost $1 \mathrm{Mb}$ in another Maori population (R Lea, personal communication).

\section{Hitchhiking variants in the $M A O-A$ gene}

Kimura ${ }^{16}$ suggested that the most prevalent form of natural selection is stabilizing selection. ${ }^{16}$ As such, it is interesting to observe that the two haplotypes observed in Maori males are also the two most 
common haplotypes found in non-Maori males. Tajima ${ }^{17}$ reported that under an infinite allele neutral mutation model, founder effects followed by a fast recovery of population size can have a strong influence on the average number of pairwise differences between chromosomes sampled from the population (that is, $\pi$ ), but little effect on the number of segregating sites (that is, $S$ ). ${ }^{17}$ In contrast, current population size has a strong influence on $S$, but not on $\pi$. The ancestors of the Maori population probably experienced repeated founder effects during migration through Polynesia, so it is therefore not surprizing that the number of unique haplotypes in Maori is low (resulting in lower $\pi$ ), while the number of segregating sites is within the range of what is observed in non-Maori groups.

The $H$ test for hitchhiking genetic variants ${ }^{18}$ is prominent in the evidence presented by Gilad et $a l^{4}$ for selection within the MAO-A gene region. ${ }^{18}$ However, this statistic requires full-sequence data (as discussed above), which we did not obtain for our samples. Given that hitchhiking has occurred within the MAO-A gene in a number of different populations (as demonstrated by Gilad et al. ${ }^{4}$ ), it is reasonable to evaluate if there has been any in the Maori population. Only two SNP haplotypes (AGCCG and GATTA) were found in Maori males (and no low-frequency variants of these were observed). One cannot conclude that the GATTA haplotype is present at an increased frequency due to selection for the AGCCG haplotype, because these two haplotypes differ at all five SNP loci. Therefore, there is no evidence for or against a hitch-hiking event when looking at these SNP variants in the Maori population, such as would be indicated by an increase in intermediate-frequency genetic variant. A loss of SNP haplotype variation such as this would be expected from a population that has experienced repeated founder effects, such as the Polynesian (Maori) population. Maintenance of low-frequency variants of these haplotypes occurs in other groups, demonstrating that low-frequency variants are unlikely to be deleterious.

The frequency of the MAO-A-uVNTR variant in Maori differs from that observed in non-Maori groups: there is a relatively low frequency of the 4-repeat VNTR allele in Maori, corresponding to a high frequency of the 3-repeat VNTR allele. If the 3-repeat VNTR allele is considered to be selectively equivalent to the 4-repeat allele, this may indicate that the more common AGCCG is under positive selective pressure.

In apparent opposition to this evidence of selection is the frequency of the 5-SNP MAO-A haplotypes in the Maori group. They do not differ significantly from those in other groups when single-mutation variants of the common SNP haplotype are included in counts of the common haplotype (Figure 3). It is reasonable to consider these extra haplotypes (GGCCG, AACCG, AGTCG and AGCTG) together with the common haplotype (AGCCG), because it would be expected that the haplotype with the largest frequency in a well-established population is older and therefore likely to accrue additional mutations over time. One of these minor variants, GGCCG, seems to be an outlier in that it is observed at a fairly high frequency in three groups typed by Gilad et al., ${ }^{4}$ namely Ashkenazi, Bedouin, Japanese and Chinese. In fact, GGCCG is the third most frequent haplotype beyond the two core haplotypes, differing from AGCCG at the first SNP in the haplotype, 1.1(263). Hence another variant has been observed at higher than expected frequency on the background of the most common SNP haplotype, AGCCG.

These two polymorphisms, MAO-A-uVNTR and 1.1(263), are separated by around 400 base pairs (Figure 1), and are both in the promoter region of the $M A O-A$ gene (about $1.5 \mathrm{~kb}$ from the $5^{\prime}$-end of the $M A O-A$ gene itself). The remainder of the polymorphic SNPs reside wholly within the $M A O-A$ gene. As mentioned previously, only demographic models and positive selection can explain an excess of high-frequency variants. ${ }^{18}$ These results appear to indicate that a specific variant of the $M A O-A$ gene, namely that described by an XGCCG SNP haplotype, is under positive selection both in Maori (as evidenced by an increased frequency of the 3-repeat VNTR allele) and non-Maori (as evidenced by an increased frequency of the GGCCG haplotype).

\section{CONCLUDING REMARKS}

The results from this analysis provide evidence that there is less genetic variation across the $M A O-A$ gene region in the Maori group compared with other groups. The individuals genotyped in this study were not known to be closely related to each other, but reduced genetic diversity in Maori populations is consistent with other studies ${ }^{19}$ - a characteristic that is predicted when considering the migration history of Maori. $^{20}$

It is clear that the Maori population studied here shows minimal variation within the $M A O-A$ gene region, most likely because the population is very new (650-750 years, or about 35 generations before present) in terms of the global history of human migration. Lack of recombination within this region (only one instance of recombination was found in 47 individuals) suggests that some positive selection may have occurred within the Maori population. A reanalysis of male haplotype data from Gilad et al. ${ }^{4}$ combined with an analysis of Maori haplotypes suggests that the gene variant described by an XGCCG haplotype has been influenced by positive selection. Full-sequence data for $M A O-A$ and large sample sizes are now required to conclusively determine whether $M A O-A$ has undergone positive selection in Polynesians. Nevertheless, our new data describe a unique demographic history of the $M A O-A$ gene in a Maori population and will be helpful for studies wishing to investigate the role of the $M A O-A$ gene in behavioral traits in Polynesians.

\section{CONFLICT OF INTEREST}

The authors declare no conflict of interest.

\section{ACKNOWLEDGEMENTS}

We wish to acknowledge and thank the participants for their invaluable contribution to this study, the Central Regional Ethics Committee for approving the study and the Wellington Health and Medical Research Foundation for helping fund the research.

1 Shih, J. C., Chen, K. \& Ridd, M. J. Monoamine oxidase: from genes to behavior. Annu. Rev. Neurosci. 22, 197-217 (1999).

2 Sabol, S. Z., Hu, S. \& Hamer, D. A functional polymorphism in the monoamine oxidase A gene promoter. Hum. Genet. 103, 273-279 (1998)

3 Ibanez, A., Perez de Castro, I., Fernandez-Piqueras, J., Blanco, C. \& Saiz-Ruiz, J. Pathological gambling and DNA polymorphic markers at MAO-A and MAO-B genes. Mol. Psychiatry 5, 105-109 (2000)

4 Gilad, Y., Rosenberg, S., Przeworski, M., Lancet, D. \& Skorecki, K. Evidence for positive selection and population structure at the human MAO-A gene. Proc. Natl Acad. Sci. USA 99, 862-867 (2002).

5 Friedlaender, J. S., Friedlaender, F. R., Reed, F. A., Kidd, K. K., Kidd, J. R., Chambers, G. K. et al. The genetic structure of Pacific islanders. PLoS Genet. 4, e19 (2008).

6 Anderson, A. The chronology of colonization in New Zealand. Antiquity 65, 767-795 (1991).

7 Marshall, S. J., Whyte, A. L. H., Hamilton, J. F. \& Chambers, G. K. Austronesian prehistory and polynesian genetics: a molecular view of human migration across the Pacific. N. Z. Sci. Rev. 62, 75-80 (2005).

8 Hall, D. A., Chambers, G. K. \& Lea, R. A. Haplotype analysis at the alcohol dehydrogenase gene region in New Zealand Maori. J. Hum. Genet. 52, 191-194 (2007).

9 Smith, T. F. \& Waterman, M. S. Identification of common molecular subsequences. J. Mol. Biol. 147, 195-197 (1981). 
10 Buetow, K. H., Edmonson, M., MacDonald, R., Clifford, R., Yip, P., Kelley, J. et al. Highthroughput development and characterization of a genomewide collection of gene-based single nucleotide polymorphism markers by chip-based matrix-assisted laser desorption/ionization time-of-flight mass spectrometry. Proc. Natl Acad. Sci. USA 98, 581-584 (2001).

11 Barrett, J. C., Fry, B., Maller, J. \& Daly, M. J. Haploview: analysis and visualization of LD and haplotype maps. Bioinformatics 21, 263-265 (2005).

12 Clark, A. G. Inference of haplotypes from PCR-amplified samples of diploid populations. Mol. Biol. Evol. 7, 111-122 (1990).

$13 \mathrm{Fu}, \mathrm{Y} . \mathrm{X} \& \mathrm{Li}, \mathrm{W}$. H. Statistical tests of neutrality of mutations. Genetics $133,693-709$ (1993).

14 Nordquist, N. \& Oreland, L. Monoallelic expression of MAO-A in skin fibroblasts. Biochem. Biophys. Res. Commun. 348, 763-767 (2006).
15 Gabriel, S. B., Schaffner, S. F., Nguyen, H., Moore, J. M., Roy, J., Blumenstiel, B. et al. The structure of haplotype blocks in the human genome. Science 296, 2225-2229 (2002).

16 Kimura, M. The neutral theory of molecular evolution: a review of recent evidence. Jap. J. Genet. 66, 367-386 (1991).

17 Tajima, F. The effect of change in population size on DNA polymorphisms. Genetics 155, 1405-1413 (1989).

18 Fay, J. C. \& Wu, C. I. Hitchhiking under positive Darwinian selection. Genetics 155, 1405-1413 (2000).

19 Shepherd, C., Harbison, S. \& Vintiner, J. Y STR haplotype data for New Zealand population groups using the Y-Plex 6 kit. Forensic Sci. Int. 145, 69-72 (2004).

20 Whittle, P. M. Health, inequality and the politics of genes. N. Z. Med. J. 123, 67-75 (2010).

Supplementary Information accompanies the paper on Journal of Human Genetics website (http://www.nature.com/jhg) 